\title{
Identification of novel putative regulators of the major apoptotic nuclease DNA Fragmentation Factor
}

\author{
Jakub Hanus, Magdalena Kalinowska-Herok and Piotr Widlak ${ }^{\bowtie}$ \\ Center for Translational Research and Molecular Biology of Cancer, Maria Sklodowska-Curie Memorial Cancer Center and Institute of Oncology, \\ Gliwice, Poland
}

\begin{abstract}
Yeast two- and three-hybrid systems were used to screen CDNA libraries from HeLa cells and human brain tissue to identify novel protein partners of DNA Fragmentation Factor, the major apoptotic nuclease. The two-hybrid system revealed the DFF45 inhibitory subunit of the nuclease as the only identified partner of the DFF40 catalytic subunit. Similar analysis revealed several protein candidates that potentially interact with the DFF45 subunit: FBXO28, FOSL1, PGK1, PCNT, FHL1 and GFAP. Recombinant GFAP protected DFF45 against cleavage with caspase- 3 and prevented activation of the DFF nuclease in vitro. In addition, three-hybrid system results revealed a putative novel protein partner of the DFF40-DFF45 heterodimer. The candidate cDNA contained two open reading frames that mapped to an intron of the GBF1 gene. Products of the candidate cDNA derived from a cell-free transcription/translation system inhibited DNA cleavage by recombinant caspase-activated DFF. This putative partner of DFF may have functional importance in regulating the apoptotic response because its RNAi silencing facilitated cleavage of the DFF45 inhibitor subunit and affected chromatin fragmentation in HeLa cells undergoing apoptosis. This hypothetical protein, named DRIG based on an acronym specifying its genomic location, could be a novel factor involved in regulation of DFF40 apoptotic nuclease.
\end{abstract}

Keywords: apoptotic nuclease, CAD, DFF, HeLa cells, yeast two-hybrid system

Received: 02 April, 2010; revised: 16 October, 2010; accepted: 22 November, 2010; available on-line: 10 December, 2010

\section{INTRODUCTION}

Genomic DNA breakdown, which was first recognized in 1980 by Wyllie (1980), is one of the hallmarks of the terminal stages of apoptosis. Such DNA breakdown occurs in two steps: first, initial cleavage into 50 - to 300-kb long genomic fragments, believed to reflect the higher-order organization of the chromatin into chromosomal loop domains (Oberhammer et al., 1993), and next, fragmentation down to oligonucleosomal-sized segments (Wyllie, 1980). Both of these cleavage events are catalyzed by the major apoptotic nuclease, DNA fragmentation factor (DFF) (Liu et al., 1997; Liu et al., 1998; Widłak, 2000), also known as caspase-activated DNase (CAD) (Enari et al., 1998). DFF40/CAD (hereafter termed DFF40) is a magnesium dependent endonuclease, both deoxyribose-specific and double-strand specific (Hanus et al., 2008), that cuts DNA to yield 5'-phosphate and 3'-hydroxyl groups, and generates ex- clusively double strand breaks (Widłak et al., 2000). In addition, the nuclease exhibits an extraordinary preference for cleaving the internucleosomal linker regions in chromatin (Widłak et al., 2000, Widłak \& Garrard, 2006). This preference possibly stems from the scissors-like structure of the DFF40 active site (Woo et al., 2004).

In the normal situation DFF40 is co-expressed with its specific inhibitor, DFF45/ICAD (hereafter termed DF45), which also acts as a molecular chaperone to correctly fold DFF40 (expression of DFF40 by itself leads only to inactive enzyme) (Enari et al., 1998; Liu et al., 1998; Halenbeck et al., 1998). The chaperone associates with DFF40 and in turn serves as an inhibitor to block its homo-oligomerization, which is required for nuclease activation (Liu et al., 1999; Widłak et al., 2003; Woo et al., 2004). In addition, a $35-\mathrm{kDa}$ splicing variant of DFF45 (DFF35/ICAD-S) also exists, which can serve as an inhibitor but not as a chaperone of DFF40 (Enari et al., 1998; Gu et al., 1999; Ageichik et al., 2007). In normal healthy cells DFF40 resides in the nucleus in the form of a heterodimer with DFF45 (Liu et al., 1998; Samejima \& Earnshaw, 1998; 2000; Lechardeur et al., 2000); complexes consisting of two DFF40 and two DFF45 molecules also have been reported (Lechardeur et al., 2005). The major activator of the DFF40 nuclease is caspase-3, which cuts DFF45 at two specific sites (Enari et al., 1998; Liu et al., 1998; Halenbeck et al., 1998). Cleavage of DFF45 results in homo-oligomerization of DFF40 (Liu et al., 1999; Widłak et al., 2003; Woo et al., 2004) followed by its effective binding to DNA (Widłak et al., 2005). Notably, it has been shown that DFF40 in association with DFF45 can also bind to DNA and be caspase-activated in the DNA-bound state (Korn et al., 2005).

Several proteins have been identified that are involved in regulating DFF nuclease in addition to DFF45 (review in: Widłak \& Garrard, 2009). These additional proteins can be divided into three functional groups: (i) factors mediating the proper folding and cellular localization of the nuclease; (ii) activators of the nuclease; and (iii) inhibitors of the nuclease. The general molecular chap-

e-mail: widlak@io.gliwice.pl

Abbreviations: CAD, caspase-activated DNase (also termed DFF40); DFF40, DNA fragmentation factor 40-kDa subunit; DFF45 DNA fragmentation factor $45-\mathrm{kDa}$ subunit; DRIG, potential DFF regulator from the intron 3 of the GBF1 gene; GBF1, Golgi specific brefeldin A resistance factor 1; GFAP, glial fibrillary acidic protein; HSP heat shock protein; ICAD, inhibitor of CAD (also termed DFF45); ORF, open reading frame; PBS, phosphate-buffered saline; SDS, sodium dodecyl sulfate; TUNEL assay, terminal deoxynucleotidyl transferase dUTP nick end labeling assay; YPDA medium, yeast peptone dextrose and adenine containing medium 
erones HSC70 and HSP40 participate in the assembly of the functional DFF heterodimer during translation (Sakahira \& Nagata, 2002); HSP70 can also stimulate and stabilize activated DFF40 (Liu et al., 2003). Furthermore, nuclear import of DFF depends on its binding to the importin $\alpha / \beta$ hetrodimer (Neimanis et al., 2007). The first identified factors that stimulate DNA cleavage by DFF were three major chromatin proteins: histone H1, HMGB1/2 and topoisomerase II (Liu et al., 1998; 1999; Widłak et al., 2000; Widłak, 2000). The C-terminal domain (CTD) of histone H1 binds to DFF40 and activates the nuclease by stimulating its ability to bind to DNA (Widłak et al., 2005). Topoisomerase II also directly interacts with DFF40 (Durrieu et al., 2000). In contrast to histone $\mathrm{H} 1$ and topoisomerase II, there is no direct interaction of HMGB1 with the nuclease; HMGB1 stimulates DNA cleavage by DFF40 through interactions of its HMG-boxes with the DNA substrate (KalinowskaHerok \& Widłak, 2008). The activities of all these three proteins apparently contribute to the specificity of chromatin cleavage by DFF: histone H1 and HMGB1 may contribute to the preferential internucleosomal cleavage that results in "DNA laddering", while topoisomerase II may be responsible for targeting the cleavage of chromosomal loop domains during the initial stages of apoptotic DNA fragmentation. Furthermore, the DFF-mediated apoptotic chromatin cleavage requires phosphorylation of histone H2A.X, which can bind DFF40 in vitro (Lu et al., 2006), although the precise mechanism of its involvement in the activation of the nuclease remains to be determined. Nuclear proteins that inhibit DFF have been identified as well. A complex of nucleophosmin/ B23 with phosphatidylinositol-3-kinases inhibits the nuclease due to its binding to activated DFF40 (but not to the DFF heterodimer) (Ahn et al., 2005). A complex of Ebp1 and AKT also inhibits the nuclease due to its binding to activated DFF40 (but it can also bind to the DFF heterodimer) (Ahn et al., 2006). In contrast, CIIA binds to the DFF heterodimer and inhibits the nuclease without affecting caspase-mediated cleavage of DFF45 (Cho et al., 2003). All these inhibitory interactions have functional importance in vivo and can suppress chromatin fragmentation in cells that have initiated apoptosis.

The aim of the present study was to identify novel proteins that interact with either the DFF heterodimer or the DFF40 and DFF45 subunits alone. To address these questions cDNA libraries from HeLa cells and human brain tissue were screened using the yeast two- and three-hybrid systems. We have identified seven new candidate DFF-interacting proteins; two of them were characterized in more details.

\section{MATERIALS AND METHODS}

Yeast two/three-hybrid systems. Full length human DFF40 cDNA (324-1340 nt, GenBank NM_004402) was inserted into the pGBKT9 vector and full length human DFF45 cDNA (99-1094 nt, GenBank NM_004401) was inserted into the pGBKT7 vector. For three-hybrid screening, cDNAs of both DFF40 and DFF45 were inserted into the pBridge vector; DFF40 was inserted into the GAL4-DB fusion site (all vectors were from Clontech). Bait plasmids were introduced into Saccharomyces cerevisiae AH109. Two commercially available cDNA libraries were used for the screening: a HeLa cell cDNA library and a human fetus brain cDNA library (cat. no. 638862 and 638869, respectively; Clontech); both librar- ies are based on the pGADT7-Rec vector in S. cerevisiae Y187. The bait strain was crossed with the library culture in $2 \times$ YPDA medium and incubated for $24 \mathrm{~h}$ at $30^{\circ} \mathrm{C}$, then harvested by centrifugation and resuspended in $0.5 \times$ YPDA medium (the yeast mating efficiency was calculated at this stage). At the first step a low stringency selection was performed by spreading the library-mating mixture on SD/-trp/-leu/-his (TDO) plates, followed by incubation until visible colonies appeared. At the second step colonies were transferred onto SD/-trp/-leu/-his/ade (QDO) plates and incubated for 8 days to ensure stronger stringency selection; only colonies that appeared on QDO medium were considered as true hybrid-positive. At the third step, Escherichia coli MG7 $\alpha$ strain was transformed with plasmid DNA isolated from hybrid yeast cells and cultured on M9/-leu medium to select for cells carrying the library vector. To identify selected cDNAs, isolated plasmid DNA was sequenced and its nucleotide sequence compared with databases.

In vitro transcription and translation. To analyze functionally the cDNAs of selected clones the TNT Quick Coupled Transcription/Translation in vitro system was used according to the manufacturer's protocol (Promega). Briefly, one microgram of library vector DNA was added to the TNT Quick Master Mix and incubated at $30^{\circ} \mathrm{C}$ for $90 \mathrm{~min}$ in a final volume of 25 $\mu \mathrm{l}$ (DNA was removed from the mixture afterward using DNase I, which was heat-inactivated later on). For analysis of specific transcripts, $10 \mu \mathrm{l}$ of the TNT reaction mixture was used as a template for cDNA synthesis using the RevertAid ${ }^{\mathrm{TM}}$ First Strand cDNA Synthesis Kit (Fermentas); cDNA synthesis was carried out with Random Hexamers primers. In addition, different amounts of the TNT reaction mixture $(1,2$ and $4 \mu \mathrm{l})$ were added to the DFF nuclease activity assay; the TNT reaction mixture was incubated with $\mathrm{RNaseA}$ to remove RNA present in the TNT mixture prior to activity assays.

The nuclease activity assay. The DFF nuclease assay was preformed as described in detail elsewhere (Widłak \& Garrard, 2006). Briefly, human recombinant DFF heterodimer (50-100 ng) was activated by incubation with an excess of caspase-3, and then $1 \mu \mathrm{g}$ of plasmid DNA was added to the caspase-activated DFF in a final volume of $20 \mu \mathrm{l}$. After incubation for $30 \mathrm{~min}$ at $33^{\circ} \mathrm{C}$ the nuclease reaction was terminated and products were separated electrophoretically on agarose gels. Alternatively, DFF was activated with caspase- 3 in the presence of the TNT reaction mixture or in the presence of human recombinant GFAP.

Cell culture. HeLa S3 cells were cultured in Dulbecco's modified Eagle's medium (Sigma) supplemented with $10 \%$ fetal bovine serum and antibiotics. Cells were maintained in a humidified incubator in a $5 \% \mathrm{CO}_{2}$ atmosphere at $37^{\circ} \mathrm{C}$. To induce apoptosis cells were incubated with $15 \mu \mathrm{M}$ cisplatin and harvested after different time periods ( 6 to $48 \mathrm{~h}$ ). Alternatively, cells were irradiated with UVC $\left(254 \mathrm{~nm} ; 50 \mathrm{~J} / \mathrm{m}^{2}\right)$ and harvested after 2, 4, 6, 8 and 24 h.

Gene silencing. Two target sequences for shRNA silencing were selected within the ORF-1 using the Ambion's siRNA Target Finder tool: s1-GATCCGGCTGCGCCCTTTATATTAG and s2-GATCCGTATCCACTGTCAAGCTGCG. The RNAi target sequences were inserted into RNAi-Ready pSiren-RetroQ vector (Clontech) using manufacturer's protocol. A negative control shRNA (oligonucleotide supplied by the manufacturer) and a positive control shRNA ( $\beta$-actin target sequence) were prepared in the same way. shRNA 
vectors were introduced into PT67 packaging cells by transfection with FuGENE HD (Roche). Virus-containing-medium from PT67 cells was used to infect HeLa cells according to Clontech's protocol. Stable transfected HeLa cell lines were selected using puromycin $(2 \mu \mathrm{g} / \mathrm{ml})$.

Transcript analysis by RT-PCR. RNA from HeLa cells was isolated using an RNA Total Mini Kit (A\&A Biotechnology), then RNA was incubated with DNase $\mathrm{I}$ and re-purified by phenol/chloroform extraction and ethanol precipitation. cDNA synthesis was carried out with an oligo $\mathrm{d}(\mathrm{T})$ primer using the RevertAid ${ }^{\mathrm{TM}}$ First Strand cDNA Synthesis Kit (Fermentas) for $60 \mathrm{~min}$ at $42^{\circ} \mathrm{C}$ followed by inactivation for $10 \mathrm{~min}$ at $70^{\circ} \mathrm{C}$. The amount of cDNA that corresponded to $100 \mathrm{ng}$ of total RNA was used as a template and 35 cycles were used for PCR reactions. Primary pairs of primers were as follows: TTGAGT'TAGCT'T'TAGTACA (forward) with GAGAAGGATAATATTGACTA (reverse), and ATCTGCCCAAGCCCTGCCAT (forward) with TTGTAGAGATGGGGGGAATC (reverse), to amplify ORF-1 and ORF-2, respectively. An additional forward primer located 600 bp upstream of ORF-1 was also used (TACCAAATTTGAAAAGAGGG). Conditions of PCR reaction (time and temperature of the annealing step) were optimized for each pair of primers.

Western blot analysis. HeLa cells were lysed in 1\% NP-40, 0.5\% sodium deoxycholate, $0.1 \%$ SDS in PBS supplemented with a protease inhibitor cocktail, and then centrifuged at $20000 \times \mathrm{g}$ for $20 \mathrm{~min}$ to remove cell remnants. Cell lysates were separated on $10 \%$ polyacrylamide/SDS gels, and then electro-transferred onto nitrocellulose membranes. Membrane-immobilized proteins were probed with rabbit anti-human DFF45 and anti-human DFF40 polyclonal antibodies (Axxora), followed by detection with peroxidase-conjugated or alkaline phosphatase-conjugated secondary antibody. Alternatively, $100 \mathrm{ng}$ of DFF45 was incubated with caspase-3 in the presence of GFAP for $30 \mathrm{~min}$ at room temperature, and then Western-blot analyzed as described above.

Analysis of chromatin cleavage in vivo. Total genomic DNA was isolated from HeLa cells by SDS/ Proteinase $\mathrm{K}$ lysis followed by phenol/chloroform extraction and ethanol precipitation, and then incubated with RNase A and separated electrophoretically on $1.5 \%$ agarose gels stained with ethidium bromide. To assess for TUNEL assay, cells were cultured and treated with $15 \mu \mathrm{M}$ cisplatin in a Lab-Tek $^{\circledR}$ II CC2 ${ }^{\mathrm{TM}}$ Chamber Slide ${ }^{\mathrm{TM}}$, then washed with $1 \times \mathrm{PBS}$ and fixed with $4 \%$ formaldehyde for $30 \mathrm{~min}$ at room temperature. Cells were extensively washed with PBS, incubated with $3 \% \mathrm{H}_{2} \mathrm{O}_{2}$ in methanol for $10 \mathrm{~min}$ at room temp. (to reduce background staining), washed again with PBS and permeabilized with $0.1 \%$ Trion X-100 in $0.1 \%$ sodium citrate for $4 \mathrm{~min}$ on ice. After washing with PBS $50 \mu$ l of the TUNEL reaction mixture (Fluorescein In Situ Cell Death Detection Kit, Roche) was added, then samples were covered with parafilm and incubated in humidified atmosphere for $90 \mathrm{~min}$ at $37^{\circ} \mathrm{C}$ in the dark. Samples were then washed extensively with PBS, stained with DAPI for $10 \mathrm{~min}$ at room temp. in the dark, washed with PBS and analyzed under a fluorescence microscope.

\section{RESULTS AND DISCUSSION}

To identify novel proteins possibly interacting with the DFF nuclease subunits we used yeast two- and threehybrid systems; the latter one, which allowed detection of tertiary protein complexes, was used to search for proteins interacting with the DFF40-DFF45 heterodimer. Two cDNA libraries of human origin were used for screening: from HeLa cells, the cells in which DFF was originally discovered (Liu et al., 1997), and from fetal brain, a tissue in which DFF is known to exhibit a clear functional importance in vivo (Slane et al., 2000). Full results of screening are shown in Table 1.

First, we searched for candidate proteins that may interact with either DFF40 or DFF45, by expressing these separately as fusion proteins with the GAL4-DNA binding (DB) domain. We found that when DFF40 was expressed as a bait protein it caused some toxicity in yeast; therefore we used the low-expression pGBKT9 vector for its expression. As expected, the screening of both libraries revealed DFF45, either full length or its splicing variant DFF35, as the major partner of DFF40. This confirmed that the DFF40-GAL4(DB) fusion protein could exhibit specific functional interactions in yeast and validated the screening approach. However, this screen did not allow identifying any other partner of DFF40, possibly because the toxicity of DFF40 when expressed alone could only be rescued by DFF45. When DFF45 was used as a bait protein several interacting proteins were identified: FBXO28 (F-box protein 28; NP_055991), FOSL1 (FOS-like antigen 1; NP_005429), PGK1 (phosphoglycerate kinase 1; NP_000282), PCNT (pericentrin; NP_006022), FHL1 (skeletal muscle LIMprotein 1; NP_001153174), and GFAP (glial fibrillary acidic protein; NP_001124491). Because none of these proteins have been reported to have clear apoptotic functions or to interact with other apoptosis-related proteins, the importance of their hypothetical interactions with DFF45 remains obscure. In fact, except for DFF40, very little is known about other proteins that interact with DFF45. Among the few proteins that have been reported to interact with DFF45 are: CIDE-B (a protein that contains oligomerization domain CIDE-N, which is present also in DFF45) (Lugovskoy et al., 1999); 14-3-3 protein (Jin et al., 2004); and DFF45 itself (Kanouchi et al., 2005). Surprisingly, our yeast two-hybrid screen did not reveal DFF40 as a partner of DFF45. Possibly, this could be explained by either toxicity of DFF40 in the library yeast strain, or by interference of the GAL4 do-

Table 1. Summary of the results of yeast two/three-hybrid screening

\begin{tabular}{lll}
\hline Bait protein & Partners identified in HeLa cell cDNA library & Partners identified in human fetal brain cDNA library \\
\hline DFF40 & DFF45 (161 clones) & DFF45 (94 clones) \\
\hline & FBXO28 (1 clone) & \\
FFS41 (1 clone) & GFAP (3 clones) \\
& PGK1 (1 clone) & FHL1 (1 clone) \\
\hline PCNT (1 clone) & $\begin{array}{ll}\text { H. sapiens chromosome 10 genome contig (2 } \\
\text { Clones) }\end{array}$ & no clone obtained \\
\hline
\end{tabular}




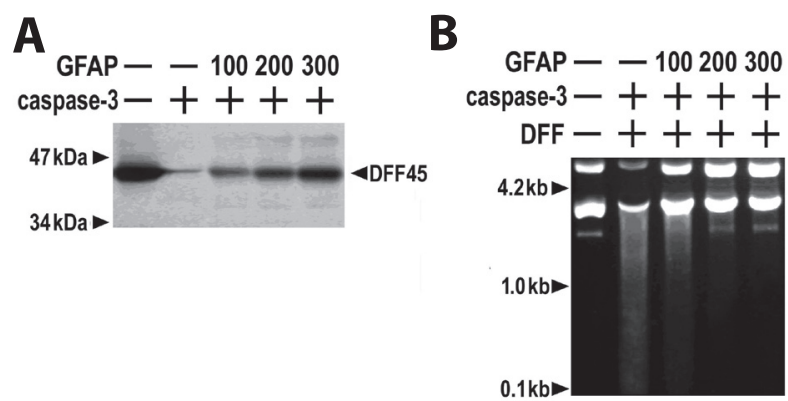

Figure 1. GFAP inhibits in vitro cleavage of DFF45 and activation of DFF nuclease by caspase-3

(A) Recombinant DFF45 was co-incubated with an excess of caspase-3 and different amounts of recombinant GFAP (ng), and then its integrity was analyzed by Western blotting. (B) DFF heterodimer was activated with caspase-3 in the presence of different amounts of GFAP (ng), and then the nuclease activity was assessed with plasmid DNA substrate in vitro.

mains with interactions between DFF40-GAL4(AD) and DFF45-GAL4(DB) fusion proteins.

We further characterized possible interactions between DFF45 and GFAP, which was identified in three clones from the human brain cDNA library. GFAP is the major astrocytic intermediate filament protein, whose malfunction is associated with the Alexander's Disease, a rare neurodegenerative disorder (Brenner et al., 2001). We assessed whether the presence of bacterially expressed human recombinant GFAP impaired caspase- 3 cleavage of DFF45 in vitro; $100 \mathrm{ng}$ of human recombinant DFF45 was co-incubated with caspase- 3 and different amounts of GFAP, and then its integrity was Western-blot analyzed. We found that GFAP inhibited DFF45 cleavage in a dose-dependent manner (Fig. 1A). In line with this observation the presence of GFAP suppressed caspase3 -catalyzed activation of DFF nuclease and the cleavage of plasmid DNA substrate in vitro (Fig. 1B). Overexpression of GFAP led to formation of pathogenic protein aggregates which triggered the stress response and resulted in the death of brain cells (Quinlan et al., 2007). In addition, cleavage of GFAP by caspase-3 was observed in degenerating astrocytes from the Alzheimer's disease brain (Mouser et al., 2006). However, no direct apoptosis-related function has been identified for this protein and thus the functional importance of its possible interactions with DFF remains unclear.

We next searched for candidate proteins that may interact with the DFF heterodimer using the so called yeast three-hybrid system; DFF40-GAL4(DB) was used

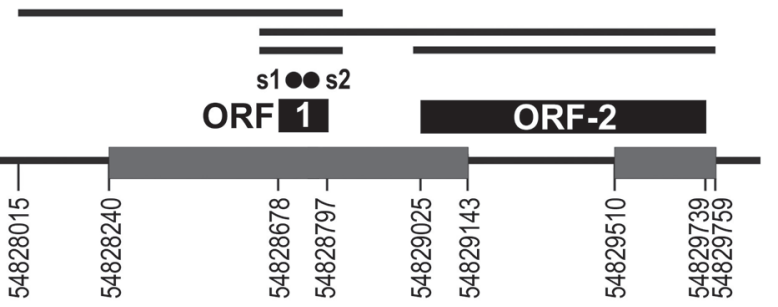

Figure 2. Structure of genomic region corresponding to hypothetical protein DRIG

Shown are sequences present in the cDNA library clone (grey boxes), hypothetical open reading frames (black boxes), shRNA target sequences (dots) and positions of major RT-PCR products (black lines). Reference positions are according to the numbering of nucleotides within GRCh37 genomic contig.

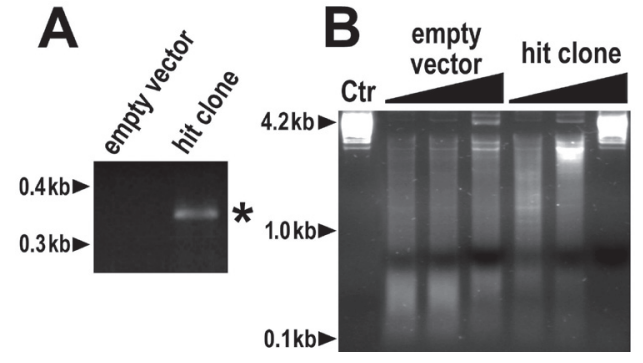

Figure 3. Influence of hypothetical protein DRIG synthesized in cell-free system in vitro on the activity of DFF nuclease

(A) RT-PCR transcript analysis of in vitro transcription/translation cell free system reaction products with control (empty vector) and cDNA of interest (hit clone corresponding to hypothetical protein DRIG); asterisk shows position of PCR product specific for ORF-2. (B) Analysis of the influence of in vitro transcription/translation reaction products on plasmid DNA cleavage by caspase-activated recombinant DFF; increasing amounts of in vitro reaction products with control (empty vector) and cDNA of interest (hit clone) were added to the nuclease assay; first lane (Ctr) represents uncleaved plasmid DNA substrate.

as a bait protein while DFF45 served as an auxiliary factor in this system. This approach revealed one hypothetical partner of the DFF heterodimer when the HeLa cell cDNA library was screened (two identical clones), and no partner when the brain cDNA library was screened (two independent screens were performed with each library). The candidate cDNA was identified by sequencing and BLAST analysis of the human genome database. The only human genomic sequence that matched the candidate cDNA $(96 \%$ identity and $98 \%$ query coverage) was located on chromosome 10 within the GRCh37 genomic contig (GenBank NT_030059) at nucleotide positions $54,828,240$ to $54,829,759$. The candidate cDNA sequence mapped to the $0.8-\mathrm{Mb}$ intron 3 of the GBF1 gene (Golgi specific brefeldin A resistance factor 1; GenBank NM_004193). The GBF1 gene is known to be ubiquitously expressed but has not been reported to have any apoptotic functions. The structure of the genomic sequence that corresponded to the candidate cDNA is shown in Fig. 2. The sequence of interest is about $1.5 \mathrm{~kb}$ in length and contains two putative open reading frames: ORF-1 (120 bp) and ORF-2 (714 bp), according to our analysis using the SPLIGN tool. ORF-2 matches two previously described expressed sequence tags: DB313950 and CV366200.1. The candidate cDNA fragment present in the library vector is shorter and lacks a $366 \mathrm{bp}$ fragment within ORF-2 (this deletion retains the phase of the reading frame). In addition, this cDNA fragment possesses 15 adenosines at its $3^{\prime}$ terminus that are not present in genomic DNA, apparently a poly(A) tail of the transcript. The sequence of interest seems to be evolutionarily conserved - it matches almost perfectly the corresponding sequence in intron 3 of the mouse Gbf1 gene ( $95 \%$ identity).

Aiming to characterize functionally the hit clone selected from the HeLa cell cDNA library we used this cDNA as a substrate in a cell-free transcription/translation system. Figure $3 \mathrm{~A}$ shows detection of transcripts specific for the analyzed sequence, which proved its transcription in this in vitro system. The in vitro transcription/translation reaction mixture was added to the reaction of caspase-3-catalyzed activation of the DFF nuclease (the mixture was pre-treated with RNase A to remove RNA that interferes with DFF activity (Widłak \& Garrard, 2006)). Figure 3B shows the effects of adding the products of the in vitro transcription/translation 


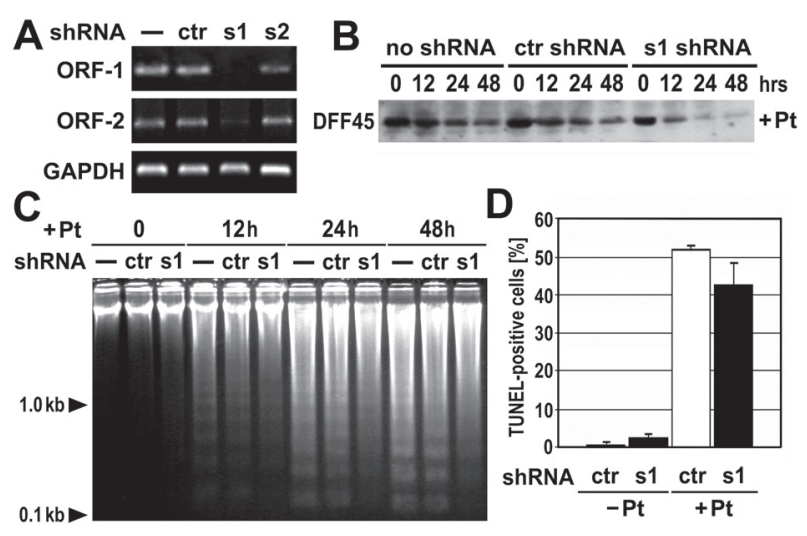

Figure 4. Effects of shRNA silencing of genomic region corresponding to hypothetical protein DRIG on apoptotic chromatin cleavage in vivo

(A) RT-PCR analysis of transcript levels of target sequences (ORF1 , ORF-2 and GAPDH control) in HeLa cells, either not transfected $(-)$ or transfected with control shRNA or ORF-1 targeted shRNA (s1 and s2) vectors. (B) Western blot analysis of DFF45 levels in cells subjected to shRNA silencing at different time points of incubation with cisplatin (+Pt) or in untreated controls (0). (C) DNA laddering in cells subjected to shRNA silencing at different time points of incubation with cisplatin (+Pt) or in untreated controls (0). (D) Assessment of TUNEL-positive fraction of cells transfected with control or S1 shRNA, and analyzed after $18 \mathrm{~h}$ of incubation with cisplatin $(+\mathrm{Pt})$ or without the drug $(-\mathrm{Pt})$. Shown are data representative for 3 biological replicas of the experiments.

reaction together with caspase-3 on plasmid DNA cleavage by DFF. We observed that products of reaction specific for the sequence present in intron 3 of GBF1 markedly inhibited DNA cleavage by DFF (components of the reaction mixture that contained empty vector showed only minor inhibitory effects). We conclude that the DNA sequence present within intron 3 of the GBF1 gene encodes a hypothetical protein that apparently affects the activity of the DFF nuclease. We named this hypothetical protein DRIG, which stands for "Potential

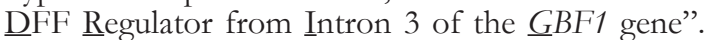

Transcription of DNA sequences corresponding to the hypothetical DRIG protein was analyzed in HeLa cells. Products of RT-PCR reactions specific for this region could be amplified using cDNA synthesized with oligo(T) primers, proving polyadenylation of transcripts, which was suggested by the composition of the sequence present in the cDNA library. Importantly, transcripts specific for the analyzed region showed very low abundance - the amount of PCR products obtained with cDNA synthesized using $100 \mathrm{ng}$ of total RNA was comparable to the amount of amplification products of $G A P D H$ gene transcripts obtained with cDNA that corresponded to $0.1-0.3 \mathrm{ng}$ of total RNA (not shown). Notably, we obtained two general types of RT-PCR products: (i) transcripts that contained both ORF-1 and ORF-2 but not sequences located upstream of ORF-1 or downstream of ORF-2, and (ii) transcripts that contained ORF-1 and sequences located up to about 600 bp upstream of ORF-1 (RT-PCR products that extended beyond this site could be detected only with cDNA synthesized using random hexamer primers); the identity of RT-PCR products was verified by sequencing. We tested different pairs of primers for RT-PCR reactions but products that contained both sequences located upstream of ORF-1 and downstream of ORF-1 (e.g., ORF-2) were not detected with cDNA synthesized using oligo(T) primers. This suggested that two types of polyadenylated transcripts are synthesized from the genomic region corresponding to hypothetical DRIG protein: ones containing both ORF-1 and ORF-2, and ones containing only ORF-1 and sequences located upstream of it (marked in Fig. 1). To further characterize transcripts corresponding to this genomic region we assessed changes in their abundance in HeLa cells treated with a pro-apoptotic factor. The amounts of RT-PCR products that corresponded to either ORF-1 or ORF-2 were analyzed in HeLa cells at different time points after UVCirradiation (from 2 to $24 \mathrm{~h}$ ). We observed that the level of ORF-1 transcripts dropped gradually after UVC-irradiation, while the level of ORF-2 transcripts decreased shortly after irradiation $(2-4 \mathrm{~h}$ ), and then increased (not shown). These observations indicate possible involvement of this genomic region in the apoptotic response and confirmed the possibility of existence of two types of transcripts arising in this region.

To further verify the importance for the apoptotic response of the genomic region corresponding to the hypothetical protein DRIG we analyzed results of its transcriptional silencing in HeLa cells. ORF-1 was selected as a target for shRNA-based silencing (which would allow inhibition of both possible types of transcripts suggested above). Two target sequences within ORF-1 were selected, s1 and s2 (see Fig. 2). Figure 4A shows that expression of the shRNA specific for the s1 sequence resulted in a marked reduction of RT-PCR products specific for both ORF-1 and ORF-2 (total inhibition of ORF-1 and about 90\% inhibition of ORF-2). The effect of this silencing on the apoptotic response was analyzed in HeLa cells treated with the genotoxic drug cisplatin; three types of cells were compared: not transfected, transfected with a control shRNA vector and transfected with the s1 shRNA vector. First we analyzed the level of DFF nuclease subunits in such cells, both untreated and treated with cisplatin. We noted some gradual decrease in the level of the DFF40 catalytic subunit after longer incubation with cisplatin, but no differences were observed between the control non-transfected cells and cells transfected with the control or the s1 shRNA vector (not shown). As expected, treatment with cisplatin resulted in the disappearance of the DFF45 inhibitory subunit. Most interestingly, however, the efficiency of DFF45 degradation was apparently higher in cells transfected with the s1 shRNA vector as compared to both controls; differences in the levels of DFF45 were not observed in the absence of cisplatin (i.e., in cells not undergoing apoptosis) (Fig. 4B).

Secondly, we analyzed whether transcriptional silencing of the genomic region corresponding to the hypothetical protein DRIG affected apoptotic chromatin cleavage induced by treatment with cisplatin; we assessed DNA laddering and TUNEL-positive cells. DNA laddering was analyzed in cells incubated with the drug for 12, 24 and $48 \mathrm{~h}$. Unexpectedly, we found that silencing of the genomic region corresponding to DRIG resulted in a slightly less advanced cisplatin-induced chromatin fragmentation (Fig. 4C). This inhibitory effect was also observed when the frequency of DNA-break-containing TUNEL-positive cells was analyzed. Cells transfected with the s1 shRNA vector were less frequently TUNEL-positive after $18 \mathrm{~h}$ of treatment with cisplatin when compared to cells transfected with control shRNA (Fig. 4D). In addition, the levels of Annexin V-positive cells and the subG1 cell fraction were analyzed to quantify apoptotic cells in our system. However, in this type of experiments we did not observe any significant effect 
of s1 shRNA on the overall number of apoptotic cells, in either normal conditions or after treatment with cisplatin (not shown). Nevertheless, our data strongly suggests that the hypothetical protein DRIG may interact with DNA Fragmentation Factor in vivo and could be involved in regulation of the apoptotic chromatin cleavage. The apparent discrepancy between the data on the cleavage of DFF45 and chromatin fragmentation could reflect the rather complex mechanism of regulation of DFF (e.g., hypothetical "cytoplasmic sequestration" of DFF in cells with down-regulated DRIG would result in both more efficient cleavage of DFF45 by cytoplasmic caspase- 3 and less intense fragmentation of nuclear chromatin).

We concluded that yeast two/three-hybrid screening of human cDNA libraries allowed identification of putative novel protein partners of the DFF nuclease. One of them was GFAP, which bound the DFF45 inhibitory subunit and inhibited its cleavage by caspase- 3 . Another candidate, whose cDNA mapped to intron 3 of the GBF1 gene, putatively interacted with the DFF heterodimer. This hypothetical protein, termed DRIG, might have functional importance because its RNAi silencing affected cleavage of DFF45 and chromatin fragmentation in HeLa cells undergoing apoptosis, and thus could be a novel factor involved in the regulation of DNA Fragmentation Factor.

\section{Acknowledgements}

We thank Professor Tomokatsu Yoshida for a generous gift of the plasmid with human GFAP cDNA, Dr. Wojciech Pigłowski for help in preparation of PT67 packaging cells, and Professor William T. Garrard for comments and help in preparation of the manuscript.

This work was supported by the Ministry of Science and Higher Education, grant N301 058 31/1763.

\section{REFERENCES}

Ageichik AV, Samejima K, Kaufmann SH, Earnshaw WC (2007) Genetic analysis of the short splice variant of the inhibitor of caspaseactivated DNase (ICAD-S) in chicken DT40 cells. J Biol Chem 282: 27374-27382.

Ahn JY, Liu X, Cheng D, Peng J, Chan PK, Wade PA, Ye K (2005) Nucleophosmin/B23, a nuclear $\mathrm{PI}(3,4,5) \mathrm{P}(3)$ receptor, mediates the antiapoptotic actions of NGF by inhibiting CAD. Mol Cell 18: $435-445$

Ahn JY, Liu X, Liu Z, Pereira L, Cheng D, Peng J, Wade PA, Hamburger AW, Ye K (2006) Nuclear Akt associates with PKC-phosphorylated Ebp1, preventing DNA fragmentation by inhibition of caspase-activated DNase. EMBO J 25: 2083-2095.

Brenner M, Johnson AB, Boespflug-Tanguy O, Rodriguez D, Goldman JE, Messing A (2001) Mutations in GFAP, encoding glial fibrillary acidic protein, are associated with Alexander disease. Nat Genet 27: $117-120$.

Cho SG, Kim JW, Lee YH, Hwang HS, Kim MS, Ryoo K, Kim MJ, Noh KT, Kim EK, Cho JH, Yoon KW, Cho EG, Park HS, Chi SW, Lee MJ, Kang SS, Ichijo H, Choi EJ (2003) Identification of a novel antiapoptotic protein that antagonizes ASK1 and CAD activities. J Cell Biol 163: 71-81.

Durrieu F, Samejima K, Fortune JM, Kandels-Lewis S, Osheroff N, Earnshaw WC (2000) DNA topoisomerase II $\alpha$ interacts with CAD nuclease and is involved in chromatin condensation during apoptotic execution. Curr Biol 10: 923-926.

Enari M, Sakahira H, Yokoyama H, Okawa K, Iwamatsu A, Nagata S (1998) A caspase-activated DNase that degrades DNA during apoptosis, and its inhibitor ICAD. Nature 391: 43-50.

Gu J, Dong RP, Zhang C, McLaughlin DF, Wu MX, Schlossman SF (1999) Functional interaction of DFF35 and DFF45 with caspaseactivated DNA fragmentation nuclease DFF40. J Biol Chem 274: 20759-20762.
Halenbeck R, MacDonald H, Roulston A, Chen T"T, Conroy L, Williams LT (1998) CPAN, a human nuclease regulated by the caspasesensitive inhibitor DFF45. Curr Biol 8: 537-540.

Hanus J, Kalinowska-Herok M, Widłak P (2008) The major apoptotic endonuclease DFF40/CAD is a deoxyribose-specific and doublestrand-specific enzyme. Apoptosis 13: 377-382.

Jin J, Smith FD, Stark C, Wells CD, Fawcett JP, Kulkarni S, Metalnikov P, O'Donnell P, Taylor P, Taylor L, Zougman A, Woodgett JR, Langeberg LK, Scott JD, Pawson T (2004) Proteomic, function$\mathrm{al}$, and domain-based analysis of in vivo 14-3-3 binding proteins involved in cytoskeletal regulation and cellular organization. Curr Biol 14: 1436-1450.

Kalinowska-Herok M, Widłak P (2008) High Mobility Group proteins stimulate DNA cleavage by apoptotic endonuclease DFF40/CAD due to HMG-box interactions with DNA. Acta Biochim Pol 55: 2126.

Kanouchi H, Nishizaki H, Minatogawa Y, Toné S. (2005) Large complex formation of the inhibitor of caspase-activated DNase. Apoptosis 10, 651-656.

Korn C, Scholz SR, Gimadutdinow O, Lurz R, Pingoud A, Meiss G (2005) Interaction of DNA fragmentation factor (DFF) with DNA reveals an unprecedented mechanism for nuclease inhibition and suggests that DFF can be activated in a DNA-bound state. $J$ Biol Chem 280: 6005-6015.

Lechardeur D, Drzymala L, Sharma M, Zylka D, Kinach R, Pacia J, Hicks C, Usmani H, Rommens JM, Luckacs GL (2000) Determinants of the nuclear localization of the heterodimeric DNA fragmentation factor (ICAD/CAD). I Cell Biol 150: 321-334.

Lechardeur D, Dougaparsad S, Nemes C, Lukacs GL (2005) Oligomerization state of the DNA fragmentation factor in normal and apoptotic cells. J Biol Chem 280: 40216-40225.

Liu X, Li P, Widłak P, Zou H, Luo X, Garrard WT, Wang X (1998) The $40-\mathrm{kDa}$ subunit of DNA fragmentation factor induces DNA fragmentation and chromatin condensation during apoptosis. Proc Natl Acad Sci USA 95: 8461-8466.

Liu QL, Kishi H, Ohtsuka K, Muraguchi A (2003) Heat shock protein 70 binds caspase-activated DNase and enhances its activity in TCRstimulated T cells. Blood 102: 1788-1796.

Liu X, Zou H, Slaughter C, Wang X (1997) DFF, a heterodimeric protein that functions downstream of caspase- 3 to trigger DNA fragmentation during apoptosis. Cell 89: 175-184.

Liu X, Zou H, Widłak P, Garrard WT, Wang X (1999) Activation of the apoptotic endonuclease DFF40 (Caspase-activated DNase or Nuclease): Oligomerization and direct interaction with histone H1. J Biol Chem 274: 13836-13840.

Lu C, Zhu F, Cho YY, Tang F, Zykova T, Ma WY, Bode AM, Dong Z (2006) Cell apoptosis: requirement of H2AX in DNA ladder formation, but not for the activation of caspase-3. Mol Cell 23: 121132.

Lugovskoy AA, Zhou P, Chou JJ, McCarty JS, Li P, Wagner G (1999) Solution structure of the CIDE-N domain of CIDE-B and a model for CIDE-N/CIDE-N interactions in the DNA fragmentation pathway of apoptosis. Cell 99: 747-755.

Mouser PE, Head E, Ha K-H, Rohn TT (2006) Caspase-mediated cleavage of glial fibillary acidic protein within degenerating astrocytes of the Alzheimer's disease brain. Am I Pathol 168: 936-947.

Neimanis S, Albig W, Doenecke D, Kahle J (2007) Sequence elements in both subunits of the DNA fragmentation factor are essential for its nuclear transport. J Biol Chem 282: 35821-35830.

Oberhammer F, Wilson JW, Dive C, Morris ID, Hickman JA, Wakeling AE, Walker PR, Sikorska M (1993) Apoptotic death in epithelial cells: cleavage of DNA to 300 and/or $50 \mathrm{~kb}$ fragments prior to or in the absence of internucleosomal fragmentation. EMBO J 12: 3679-3684.

Quinlan RA, Brenner M, Goldman JE, Messing A (2007) GFAP and its role in Alexander Disease. Exp Cell Res 313: 2077-2087.

Sakahira H, Nagata S (2002) Co-translational folding of caspase-activated DNase with Hsp70, Hsp40, and inhibitor of caspase-activated DNase. I Biol Chem 277: 3364-3370.

Samejima K, Earnshaw WC (1998) ICAD/DFF regulator of apoptosis is nuclear. Exp Cell Res 243: 453-459.

Samejima K, Earnshaw WC (2000) Differential localization of ICAD$\mathrm{L}$ and ICAD-S in cells due to removal of a C-terminal NLS from ICAD-L by alternative splicing. Exp Cell Res 255: 314-320.

Slane JM, Lee HS, Vorhees CV, Zhang J, Xu M (2000) DNA fragmentation factor 45 deficient mice exhibit enhanced spatial learning and memory compared to wild-type control mice. Brain Res 867: 70-79.

Widłak P (2000) DFF40/CAD hypersensitive sites are potentially involved in high molecular weight DNA fragmentation during apoptosis. Cell Mol Biol Lett 5: 373-379.

Widłak P, Garrard W'T (2006a) The apoptotic endonuclease DFF40/ CAD is inhibited by RNA, heparin and other polyanions. Apoptosis 11: $1331-1337$.

Widłak P, Garrard W'T (2006b) Unique features of the apoptotic endonucleaase DFF40/CAD relative to micrococcal nuclease as a structural probe for chromatin. Biochem Cell Biol 84: 405-410. 
Widłak P, Li P, Wang X, Garrard W'T (2000) Cleavage preferences of the apoptotic endonuclease DFF40 (Caspase-activated DNase or Nuclease) on naked DNA and chromatin substrates. I Biol Chem 275: 8226-8232

Widłak P, Lanuszewska J, Cary RB, Garrard WT (2003) Subunit structures and stoichiometries of human DFF proteins before and after induction of apoptosis. J Biol Chem 278: 26915-26922.

Widłak P, Kalinowska M, Parseghian MH, Lu X, Hansen JC, Garrard WT (2005) The histone H1 C-terminal domain binds to the apoptotic nuclease, DNA Fragmentation Factor (DFF40/CAD) and stimulates DNA cleavage. Biochemistry 44: 7871-7878.
Widłak P, Garrard WT (2009) Roles of the major apoptotic nuclease DNA Fragmentation Factor — in biology and disease. Cell Mol Life Sci 66: 263-274.

Woo EJ, Kim YG, Kim MS, Han WD, Shin S, Robinson H, Park SY, Oh BH (2004) Structural mechanism for inactivation and activation of CAD/DFF40 in the apoptotic pathway. Mol Cell 14: 531-539.

Wyllie AH (1980) Glucocorticoid-induced thymocyte apoptosis is associated with endogenous endonuclease activation. Nature 284: 555-556. 